\title{
Multivariate Skew Distributions Based on the GT-Copula*
}

\author{
Beatriz Vaz de Melo Mendes ${ }^{* *}$ \\ Olcay Arslan ${ }^{* * *}$
}

\begin{abstract}
In this paper we obtain the expression for the copula pertaining to the multivariate generalized $t$ distribution, the generalized $t$-copula, or in short, GT-copula. The GT-copula generalizes some well known copulas, such as the one associated with the multivariate $t$ distribution ( $t$-copula). We derive the expression for its tail dependence coefficient and show that the GT-copula may adjust for stronger tail dependence when compared to the $t$-copula. The potentiality of the GT-copula allows for modeling varying degrees and different types (linear and nonlinear) of dependence, as well as multimodality. By applying Sklar's theorem to the GT-copula, and by mixing symmetric and asymmetric margins, we construct a new family of multivariate distributions. The resulting distributions are suitable for modeling a wide variety of skew datasets. The flexibility of the copula approach suggests applications in many fields, such as environment and finance. We provide an illustration, where we quantify stock market linkages.
\end{abstract}

Keywords: Copulas, GT Distribution, Skew GT Distribution, Skew Meta-Elliptical Distributions, Financial Markets Interdependencies.

JEL Codes: C12, C13, C14, C52.

\footnotetext{
${ }^{*}$ Submitted in January 2006. Revised in September 2006. The authors wish to thank the Editor and the two referees for their valuable comments and suggestions, which have greatly improved the paper. Beatriz Mendes was partially supported by CNPq-Brazil - Project $478712 / 01$ 2, and Convênio MCT/CNPq/FAPERJ - Edital 2004, Cópulas: Permutabilidade, Assimetrias, Inferência Estatística.

${ }^{* *}$ Statistics Department, Federal University at Rio de Janeiro, Brazil. E-mail: beatriz@im.ufrj.br

${ }^{* * *}$ Statistics Department, Cukurova University, Turkey.
}

Brazilian Review of Econometrics

v. $26, \mathrm{n}^{\circ} 2$, pp. $235-255$ Nov 2006 


\section{Introduction}

McDonald and Newey (1988) introduced the univariate GT Distribution as an alternative to the normal and $t$ distributions when modeling errors in regression. The GT family applies to symmetric, fat-tailed data and therefore adjusts for the leptokurtosis of nonnormal data. Recently, Theodossiou (1998) introduced the univariate skew GT Distribution and Arslan (2004) defined a new family of multivariate generalized $t$ (MGT) distributions. The MGT family includes as special cases the multivariate $t$ distribution, and the generalized $t$ distribution defined by Arellano-Valle and Bolfarine (1995).

In this paper we obtain the expression for the copula pertaining to the MGT family of distributions, the GT-copula, and investigate its properties. The GTcopula may be seen as a generalization of the well known copula associated with the multivariate $t$ distribution ( $t$-copula). We derive the expressions of the copulabased coefficients of upper and lower tail dependence $\left(\lambda_{U}\right.$ and $\left.\lambda_{L}\right)$, and show that the GT-copula may possess stronger tail dependence when compared to the $t$ copula.

One useful application of copulas is the construction of multivariate distributions using Sklar's theorem (Sklar, 1959). Given the marginals, many interesting multivariate distributions, ranging from symmetric to asymmetric, can be obtained from the GT-copula. A similar technique was used by Fang et al. (2002), where they proposed the family of meta-elliptical distributions. We provide some other examples of this type of family, combining the GT-copula with GT and skew GT marginals.

The flexibility of the copula approach (which separately models margins and dependence structure) allows for modeling many different data types, for example, skew and multimodal data. In addition, the potentiality of the GT-copula allows for modeling different degrees and types (linear and nonlinear) of dependence. The combination of these two properties suggests applications in fields such as environment, insurance, and finance. In finance, the GT-copula may be used, for example, to model dependent risks and to compute measures of interdependence and contagion.

We provide an illustration in finance using pairs of stock market indexes. Whenever there exists positive association between two markets, there exists contagion, and the asymptotic interdependence may be severely underestimated if a copula with zero tail dependence coefficient is assumed. To measure linkages at high quantiles between markets, we define a selection of tail events involving the unconditional model based Value-at-Risk with small exceedance probability in each margin, and compute their probabilities. These numbers may provide new insights on the interrelationships of these markets. 
The dynamics in mean and volatility of the series may affect the copula and dependence measures estimates. To investigate this issue, we model the conditional mean and volatility using time series and GARCH models, and fit copulas to the filtered data.

The remainder of this paper is organized as follows. In Section 2 we briefly review the concept of copula and define some dependence measures. In Section 3 we obtain the copula of the MGt distribution and derive its properties. In Section 4 we provide some examples of the construction of new families of multivariate skew distributions and give an algorithm to generate from the GT-copula. In Section 5 we illustrate the usefulness of the copula approach in finance, and compute market linkages using raw and filtered log-returns. In Section 6 we discuss our results and conclude.

\section{Copulas}

\subsection{Definition of copula}

Let $F$ be the absolutely continuous distribution function (cdf) of the random vector $\boldsymbol{X}=\left(X_{1}, \ldots, X_{d}\right) \in \Re^{d}$. Let $F_{1}, \ldots, F_{d}$ be the marginal cdfs, and let $X_{i} \mapsto$ $F_{i}\left(X_{i}\right)=U_{i}, i=1, \ldots, d$ be the probability integral transformation to the standard uniform distribution. The copula $C_{F}$ pertaining to $F$ is defined as

$$
C_{F}\left(u_{1}, \ldots, u_{d}\right)=F\left(F_{1}^{-1}\left(u_{1}\right), \ldots, F_{d}^{-1}\left(u_{d}\right)\right), \quad \forall\left(u_{1}, \ldots, u_{d}\right) \in(0,1)^{d}
$$

where $F_{i}^{-1}$, is the quantile function of $F_{i}, i=1, \ldots, d$, that is, $F_{i}^{-1}(p)=\inf \left\{x \mid F_{i}(x)\right.$ $\geq p\}, p \in(0,1)$.

The copula $C_{F}$ completely specifies the distribution $F$ (Sklar's theorem, Sklar (1959)) since $\forall \boldsymbol{x}=\left(x_{1}, \ldots, x_{d}\right) \in \Re^{d}$ :

$$
F\left(x_{1}, \ldots, x_{d}\right)=C_{F}\left(F_{1}\left(x_{1}\right), \ldots, F_{d}\left(x_{d}\right)\right)
$$

From (1) and (2) we observe that the copula $C_{F}$ is the cdf of the random vector $\left(U_{1}, \ldots, U_{d}\right)$ and contains all information about the dependence structure existing among the margins $F_{i}$ 's. The copula representation of $F$ is unique when all $F_{i}$, $i=1, \ldots, d$ are continuous Sklar (1959). Next, we provide the densities of two important families of elliptical copulas.

(i) Density of the Gaussian Copula $C_{G a}^{R}$ :

$$
c_{G a}^{R}\left(u_{1}, \ldots, u_{d}\right)=|R|^{-1 / 2} \exp \left\{\frac{1}{2} \mathbf{z}^{\prime}\left(I_{d}-R^{-1}\right) \mathbf{z}\right\},\left(u_{1}, \ldots, u_{d}\right) \in(0,1)^{d}
$$

where $\mathbf{z}=\left(z_{1}, \ldots, z_{d}\right)$ with $z_{i}=\Phi^{-1}\left(u_{i}\right)$ are the standard normal quantiles, $\Phi$ represents the (univariate) cdf of a standard normal random variable, and $R$ is the cor- 
relation matrix. The Gaussian copula is the copula pertaining to a $d$-variate normal distribution (with cdf $\Phi_{d}^{R}$ ) with standard normal margins and (Pearson) correlation matrix $R$. From $(2), F\left(x_{1}, \ldots, x_{d}\right)=\Phi_{d}^{R}\left(x_{1}, \ldots, x_{d}\right)=C_{G a}^{R}\left(\Phi\left(x_{1}\right), \ldots, \Phi\left(x_{d}\right)\right)$. (ii) Density of the t-copula $C_{t}^{R, \nu}$ :

$$
c_{t}^{R, \nu}\left(u_{1}, \ldots, u_{d}\right)=\frac{K^{*}|R|^{-1 / 2}\left(1+\frac{\mathbf{z}^{\prime} R^{-1} \mathbf{z}}{\nu}\right)^{-\frac{\nu+d}{2}}}{\prod_{i=1}^{d} f_{\nu}\left(t_{\nu}^{-1}\left(u_{i}\right)\right)},\left(u_{1}, \ldots, u_{d}\right) \in(0,1)^{d}
$$

where $K^{*}=\frac{\Gamma\left(\frac{\nu+d}{2}\right)(\pi \nu)^{-\frac{d}{2}}}{\Gamma\left(\frac{\nu}{2}\right)}, \mathbf{z}=\left(z_{1}, \ldots, z_{d}\right)$, with $z_{i}=t_{\nu}^{-1}\left(u_{i}\right)$, and where $t_{\nu}^{-1}(\cdot)$ represents the quantile function of a (univariate) standard $t$-distributed random variable with $\nu$ degrees of freedom (d.f.) with density function $f_{\nu}$ and cdf $t_{\nu}$. The $t$-copula depends only on $R$ and $\nu$, and the value for $\nu$ is common for marginal and joint distributions. The $t$-copula is the copula pertaining to a $d$-variate $t$ distribution with $\nu$ d.f. and correlation matrix $R$. A detailed study of its properties may be found in Demarta and McNeill (2004).

\subsection{Copula-based dependence measures}

The scale-invariant dependence structure of a multivariate distribution $F$ is best represented by its copula, which remains unchanged when the marginal variables are subjected to strictly increasing transformations. Some dependence measures possess this invariance property and may be conveniently represented using the corresponding $C_{F}$ copula. Examples of such copula-based dependence measures are Kendall's $\tau$ and Spearman's $\rho$.

An important example of a dependence measure not sharing the invariance property is the Pearson correlation coefficient $\rho$, the canonical measure in the elliptical world. It can be shown (Joag-Dev et al., 1983) that the Pearson correlation coefficient $\tilde{\rho}_{i j}$ between the margins $i$ and $j$ of the copulas $C_{G a}^{R}$ and $C_{t}^{R, \nu}$ are slightly smaller than the $\rho_{i j}$ of the corresponding distributions. Next we give the definitions of Kendall's $\tau$ and Spearman's $\rho$ along with the concept of tail dependence. For the sake of simplicity, we write $X_{1}$ and $X_{2}$ representing any $(i, j)$ pair of margins.

Kendall's $\tau\left(X_{1}, X_{2}\right)$ is defined as

$$
\tau\left(X_{1}, X_{2}\right)=\operatorname{Pr}\left\{\left(X_{1}-\tilde{X}_{1}\right)\left(X_{2}-\tilde{X}_{2}\right)>0\right\}-\operatorname{Pr}\left\{\left(X_{1}-\tilde{X}_{1}\right)\left(X_{2}-\tilde{X}_{2}\right)<0\right\}
$$

where $\left(X_{1}, X_{2}\right)$ and $\left(\tilde{X}_{1}, \tilde{X}_{2}\right)$ are independent random vectors from $F$. Joe $(1997)$ and Nelsen (1999) provide the copula-based expression for $\tau$ :

$$
\tau\left(X_{1}, X_{2}\right)=4 \iint_{[0,1]^{2}} C_{F}\left(u_{1}, u_{2}\right) d C_{F}\left(u_{1}, u_{2}\right)-1
$$


Expression (6) may be rewritten as $\tau\left(X_{1}, X_{2}\right)=4 E\left[C_{F}\left(U_{1}, U_{2}\right)\right]-1$, where $\left(U_{1}, U_{2}\right)$ $\sim C_{F}$. They make clear the role of the copula as a function that summarizes the dependence structure of any joint distribution, independently of the choice of marginals. A multivariate extension of Kendall's $\tau$ is derived in Jouini and Clemen (1996):

$$
\tau\left(X_{1}, \ldots, X_{d}\right)=\frac{1}{2^{d-1}-1}\left[2^{d} \int \ldots \int_{[0,1]^{d}} C_{F}\left(u_{1}, \ldots, u_{d}\right) d C_{F}\left(u_{1}, \ldots, u_{d}\right)-1\right]
$$

Spearman's $\rho_{S}\left(X_{1}, X_{2}\right)$ is the Pearson's $\rho$ of $\left(F_{1}\left(X_{1}\right), F_{2}\left(X_{2}\right)\right)$ and is defined as

$$
\begin{aligned}
\rho_{S}\left(X_{1}, X_{2}\right) & =3 \\
\left(\operatorname{Pr}\left\{\left(X_{1}-\tilde{X}_{1}\right)\left(X_{2}-\underline{X}_{2}\right)>0\right\}\right. & \left.-\operatorname{Pr}\left\{\left(X_{1}-\tilde{X}_{1}\right)\left(X_{2}-\underline{X}_{2}\right)<0\right\}\right)
\end{aligned}
$$

where $\left(X_{1}, X_{2}\right),\left(\tilde{X}_{1}, \tilde{X}_{2}\right)$, and $\left(\underline{X}_{1}, \underline{X}_{2}\right)$ are independent random vectors from $F$. The copula-based expression for $\rho_{S}$ is (Joe (1997) and Nelsen (1999)):

$$
\rho_{S}\left(X_{1}, X_{2}\right)=12 \iint_{[0,1]^{2}} C_{F}(u, v) d u d v-3
$$

A different copula-based measure of association is the so-called tail dependence coefficient. If there exists positive association (Tawn (1988), Coles et al. (1999)) between extreme events of $X_{1}$ and $X_{2}$, then the conditional probability

$$
\lambda_{U}(\alpha)=\operatorname{Pr}\left\{X_{1}>F_{1}^{-1}(1-\alpha) \mid X_{2}>F_{2}^{-1}(1-\alpha)\right\}
$$

is greater than zero and decreases with $\alpha$. The upper tail dependence coefficient $\lambda_{U}$ is the limit of the function $\lambda_{U}(\alpha)$, as $\alpha \rightarrow 0^{+}$, if this limit exists. The two variables $X_{1}$ and $X_{2}$ are said to be asymptotically dependent in the upper tail if $\lambda_{U} \in(0,1]$, and asymptotically independent if $\lambda_{U}=0$. The lower tail dependence coefficient $\lambda_{L}$ is defined similarly.

As shown in Joe (1997), both the upper and the lower tail dependence coefficients can be expressed using the copula $C_{F}$ pertaining to $\left(X_{1}, X_{2}\right)$ :

$$
\lambda_{U}=\lim _{u \uparrow 1} \frac{\bar{C}_{F}(u, u)}{1-u}
$$

where $\bar{C}_{F}\left(u_{1}, u_{2}\right)=\operatorname{Pr}\left\{U_{1}>u_{1}, U_{2}>u_{2}\right\}$, and

$$
\lambda_{L}=\lim _{u \downarrow 0} \frac{C_{F}(u, u)}{u}
$$


if these limits exist. There is lower tail dependence between the variables if $\lambda_{L} \in$ $(0,1]$. Lower tail independence corresponds to $\lambda_{L}=0$.

Closed forms for $\lambda_{U}$ and $\lambda_{L}$ are not available for all copulas. For the copula of a bivariate elliptically symmetric distribution these two measures coincide. Let $\lambda$ represent the common measure. Embrechts et al. (2002) showed that

$$
\lambda=2 \lim _{x \downarrow-\infty} \operatorname{Pr}\left\{X_{2} \leq x \mid X_{1}=x\right\}
$$

For example, $\lambda=2 \lim _{x \rightarrow \infty} \bar{\Phi}\left(\frac{x \sqrt{1-\rho}}{\sqrt{1+\rho}}\right)=0$ in the case of a bivariate Gaussian copula with correlation coefficient $\rho$, and where $\bar{\Phi}(x)=1-\Phi(x)$; and $\lambda=2 \bar{t}_{\nu+1}\left(\frac{\sqrt{\nu+1} \sqrt{1-\rho}}{\sqrt{1+\rho}}\right)$, where $\bar{t}_{\nu}(x)=1-t_{\nu}(x)$, in the case of a bivariate $t$ copula.

\section{The GT-copula}

Let $\boldsymbol{X}$ be a $d$-dimensional random vector with an MGT $(\mu, \Sigma, \lambda, \beta, q)$ distribution (see Arslan (2004) for details). The density function of $\boldsymbol{X}$ is

$$
f(\boldsymbol{x} ; \mu, \Sigma, \lambda, \beta, q)=K \lambda^{-d / 2}|\Sigma|^{-1 / 2} \frac{1}{\left\{2 q+\left(\frac{s}{\lambda}\right)^{\beta}\right\}^{q+\frac{d}{2 \beta}}}
$$

where

$$
K=\frac{\beta \Gamma\left(\frac{d}{2}\right) \Gamma\left(q+\frac{d}{2 \beta}\right)(2 q)^{q}}{\pi^{\frac{d}{2}} \Gamma(q) \Gamma\left(\frac{d}{2 \beta}\right)}=\frac{\beta \Gamma\left(\frac{d}{2}\right)(2 q)^{q}}{\pi^{\frac{d}{2}} B\left(q, \frac{d}{2 \beta}\right)}
$$

is the normalizing constant, $s=(\boldsymbol{x}-\mu)^{\prime} \Sigma^{-1}(\boldsymbol{x}-\mu)$ and $B(\cdot, \cdot)$ denotes the beta function. Here, $\beta>0$ and $q>0$ are shape parameters, $\mu \in \Re^{d}$ is the location parameter, $\Sigma$ is a positive definite scatter matrix, and we set the scale parameter $\lambda=1$. The parameters $\beta$ and $q$ both control the shape of the density. Larger values of $\beta$ and $q$ yield an MGT density with thinner tails, and smaller values of $\beta$ and $q$ are associated with thicker tailed densities. Although the parameters $\beta$ and $q$ play similar roles, the parameter $\beta$ has larger influence on the density shape and affects the kurtosis. More information on the MGt distribution can be found in Arslan (2004).

The MGT is a family of elliptical distributions with density generator function

$$
\psi(t)=K\left\{2 q+t^{\beta}\right\}^{-\left(q+\frac{d}{2 \beta}\right)}
$$

It can be shown (Arslan, 2004) that the random vector $\boldsymbol{X}$ has the stochastic representation 


$$
X=\mu+V A^{\prime} U^{(d)}
$$

where $V$ is an absolutely continuous random variable with a generalized beta distribution of second kind, $U^{(d)}$ is a random variable uniformly distributed on the unit sphere in $\Re^{d}$, and $V$ and $U^{(d)}$ are independent.

We now obtain the GT-copula pertaining to the MGt distribution. For simplicity we write it in the bivariate case. The extension to the multivariate case is straightforward.

Let $\boldsymbol{Z}=\left(Z_{1}, Z_{2}\right)^{\prime} \sim \operatorname{MGT}(0, R, 1, \beta, q)$ with $R=\left(\begin{array}{cc}1 & \rho \\ \rho & 1\end{array}\right)$, where $-1<\rho<1$. The density function and the cdf of $\boldsymbol{Z}$ are, respectively,

$$
f\left(z_{1}, z_{2}, \rho, \beta, q\right)=K \frac{1}{\sqrt{1-\rho^{2}}}\left[2 q+\left(\frac{z_{1}^{2}+z_{2}^{2}-2 \rho z_{1} z_{2}}{1-\rho^{2}}\right)^{\beta}\right]^{-q-\frac{1}{\beta}}
$$

and $F\left(z_{1}, z_{2}, \rho, \beta, q\right)=\int_{-\infty}^{z_{1}} \int_{-\infty}^{z_{2}} f\left(z_{1}, z_{2}, \rho, \beta, q\right) d z_{2} d z_{1}$. The marginal density function and cdf of $Z_{i}$, for $i=1,2$, are given by

$$
q(z, \beta, q)=K \int_{z^{2}}^{\infty}\left(y-z^{2}\right)^{-1 / 2}\left[2 q+y^{\beta}\right]^{-q-\frac{1}{\beta}} d y
$$

and

$$
Q(z, \beta, q)=\frac{1}{2}+\int_{z^{2}}^{\infty} \arcsin \left(\frac{z}{\sqrt{y}}\right)\left[2 q+y^{\beta}\right]^{-q-\frac{1}{\beta}} d y+\frac{\pi}{2} \int_{0}^{z^{2}}\left[2 q+y^{\beta}\right]^{-q-\frac{1}{\beta}} d y
$$

We refer to Fang et al. (2002) and Fang et al. (2005) for the expression of the marginal density and the distribution function for any elliptical distribution.

Using the copula definition (1) we obtain the bivariate GT-copula

$$
\begin{aligned}
C_{G T}^{\rho, \nu}\left(u_{1}, u_{2}\right)= & \int_{-\infty}^{Q^{-1}\left(u_{1}\right)} \int_{-\infty}^{Q^{-1}\left(u_{2}\right)} \frac{K}{\sqrt{1-\rho^{2}}} \\
& \left\{\nu+\left(\frac{s^{2}-2 \rho s t+t^{2}}{\left(1-\rho^{2}\right)}\right)^{\beta}\right\}^{-\nu / 2-1 / \beta} d s d t
\end{aligned}
$$

where $2 q=\nu$. When $\beta=1$ we get the bivariate $t$-copula (see (4)). The density function of the bivariate GT-copula is given by 


$$
\begin{aligned}
& c_{G T}^{\rho, \nu}\left(u_{1}, u_{2}\right)= \\
& \frac{K \frac{1}{\sqrt{1-\rho^{2}}}\left[\nu+\left(\frac{z_{1}^{2}+z_{2}^{2}-2 \rho z_{1} z_{2}}{1-\rho^{2}}\right)^{\beta}\right]^{-\frac{\nu}{2}-\frac{1}{\beta}}}{\left[\int_{z_{1}^{2}}^{\infty}\left(y-z_{1}^{2}\right)^{-1 / 2}\left[\nu+y^{\beta}\right]^{-\frac{\nu}{2}-\frac{1}{\beta}} d y\right]\left[\int_{z_{2}^{2}}^{\infty}\left(y-z_{2}^{2}\right)^{-1 / 2}\left[\nu+y^{\beta}\right]^{-\frac{\nu}{2}-\frac{1}{\beta}} d y\right]}
\end{aligned}
$$

where $z_{i}=Q^{-1}\left(u_{i}\right)$, for $i=1,2$. Since the integrations in (20) are not tractable, we cannot obtain the explicit formula for the density function of the GT-copula. Note that as $\rho \rightarrow+1$ or $\rho \rightarrow-1$, the GT-copula approaches, respectively, the Fréchet-Hoeffding upper and lower bounds copulas of perfect dependence (Joe, 1997).

The density function of the $d$-dimensional GT-copula is given by

$$
c_{G T}^{R, \nu}\left(u_{1}, \ldots, u_{d}\right)=\frac{K|R|^{-1 / 2}\left[\nu+\left(\mathbf{z}^{\prime} R^{-1} \mathbf{z}\right)^{\beta}\right]^{-\frac{\nu}{2}-\frac{d}{2 \beta}}}{I_{1} \cdots I_{d}}
$$

where $\mathbf{z}=\left(z_{1}, \ldots, z_{d}\right)$, and, for $j=1, \ldots, d, I_{j}=\int_{z_{j}^{2}}^{\infty}\left(y-z_{j}^{2}\right)^{-1 / 2}\left[\nu+y^{\beta}\right]^{-\frac{\nu}{2}-\frac{1}{\beta}} d y$.

The coefficients $\tau$ and $\rho_{S}$ of the GT-copula are obtained by using (19) and (20) in (6) and (8). However, it is important to emphasize that although all degrees of dependence are captured by the new proposed family of copulas as $\tau$ or $\rho_{S}$ range between -1 and $1, \tau=\rho_{S}=0$ does not correspond to stochastic independence.

To compute the tail dependence coefficients $\lambda_{L}$ and $\lambda_{U}$ of the GT-copula we consider the random vector $\boldsymbol{Z}=\left(Z_{1}, Z_{2}\right)^{\prime} \sim \operatorname{MGT}(0, R, 1, \beta, q)$ with the copula $C_{G T}^{\rho, \nu}\left(Q\left(z_{1}, \beta, q\right), Q\left(z_{2}, \beta, q\right)\right)$. The conditional distribution of $Z_{1} \mid Z_{2}=z$ is an elliptically contoured distribution with expected value and variance given by

$$
E\left(Z_{1} \mid Z_{2}=z\right)=\rho z \text { and } \operatorname{Var}\left(Z_{1} \mid Z_{2}=z\right)=g(z)\left(1-\rho^{2}\right)
$$

where $g(z)$ is a positive real valued function. The conditional distribution function of $Z_{1} \mid Z_{2}=z$ is

$$
Q^{*}(z, \beta, q)=K_{1} \int_{-\infty}^{z}\left(1-\rho^{2}\right)^{-1 / 2}\left(2 q+\left(z^{2}+\frac{\left(z_{1}-\rho z\right)^{2}}{1-\rho^{2}}\right)^{\beta}\right)^{-q-\frac{1}{\beta}} d z_{1}
$$

where $K_{1}=\frac{q^{q+\frac{1}{\beta}} \Gamma\left(\frac{1}{2}\right)}{\pi^{\frac{1}{2}} g_{1}(z, \beta, q)}$ and $g_{1}(z, \beta, q)$ is some positive function (see Arslan (2004)). Since the GT-copula is a symmetric copula, and following Embrechts et al. (2002), to derive the tail dependence coefficients, we apply the quantile transformation of (18) on equation (11) and obtain 


$$
\begin{aligned}
\lambda_{U} & =2 \lim _{z \rightarrow \infty} \operatorname{Pr}\left\{Z_{1}>z \mid Z_{2}=z\right\} \\
& =2\left(1-\lim _{z \rightarrow \infty} \operatorname{Pr}\left\{Z_{1} \leq z \mid Z_{2}=z\right\}\right) \\
& =2 \lim _{z \rightarrow \infty} \bar{Q}^{*}\left(\frac{z}{\sqrt{g(z)}} \frac{\sqrt{1-\rho}}{\sqrt{1+\rho}}\right)
\end{aligned}
$$

For elliptical distributions, an expression for the tail dependence coefficient was derived independently at the same time by Schmidt (2002) and Hult and Lindskog (2002), see also Frahm et al. (2003) and Frahm et al. (2005). It was shown that if the generator variable $V$ (see (15) in the case of the MGt distribution) is regularly varying with tail index $\xi>0$ (a measurable function $\phi$ from $\Re_{+} \rightarrow \Re_{+}$is regularly varying at $\infty$ with tail index $\xi \in \Re$, if for any $\left.a>0, \lim _{t \rightarrow \infty} \frac{\phi(a t)}{\phi(t)}=a^{-\xi}\right)$, then for all $i \neq j$,

$$
\lambda_{U}\left(X_{i}, X_{j}\right)=\lambda_{L}\left(X_{i}, X_{j}\right)=\frac{\int_{\left(\pi / 2-\arcsin \rho_{i j}\right) / 2}^{\pi / 2} \cos ^{\xi} t d t}{\int_{0}^{\pi / 2} \cos \xi t d t}
$$

where the linear correlation coefficient $\rho_{i j}$ is an extension of the usual definition of the Pearson correlation coefficient, which allows for its interpretation as a scalar measure of dependence that should not rely on the finiteness of certain moments. As noted by Hult and Lindskog (2002), from (23) we observe that we can have a coefficient of tail dependence significantly larger than zero even if $\rho_{i j}$ is zero or negative.

As pointed out by a referee, from the relation between the non-negative random variable $V$ and the generator $\psi$ given in (14), it is possible to obtain the expression for tail dependence of an MGt distribution. For $q=\nu / 2$ we have

$$
\psi(t) \propto K\left(\nu+t^{\beta}\right)^{-\left(\frac{\nu}{2}+\frac{d}{2 \beta}\right)} \sim t^{-\left(\frac{\beta \nu}{2}+\frac{d}{2}\right)}
$$

and thus the density function of the generating variate has tail index $\beta \nu+1$, and hence the generating variable $V$ possesses tail index $\xi=\beta \nu$ (for details about the relation between $V$ and the generator function, see Fang et al. (1990), Schmidt (2002), and also Frahm et al. (2003)). By using the same arguments in Schmidt (2002) and Hult and Lindskog (2002) we obtain the tail dependence coefficient for the MGT distribution:

$$
\lambda=2 \bar{t}_{\beta \nu+1}\left(\frac{\sqrt{\beta \nu+1} \sqrt{1-\rho}}{\sqrt{1+\rho}}\right)
$$

The tail dependence of a GT-copula is decreasing in $\nu=2 q$ and $\beta$, and increasing in $\rho$. For $\rho<1, \lambda_{U}$ and $\lambda_{L}$ go to zero as $\nu \rightarrow \infty$. When $\beta=1$ we obtain 
the tail dependence of a $t$-copula. For fixed $\nu$ and $\rho$, greater tail dependence is obtained by small values of $\beta$, since as $\beta$ decreases, the MGT gets fatter tails.

There is now a vast literature on how to estimate tail dependence indexes. Among them we cite Wendin (2002), who proposed an estimator for the tail dependence coefficient based on an estimator of a measure $\mu$, which may alternatively express the notion of multivariate regular variation. Schmidt and Stadtmüller (2003) introduced the concept of tail copulas and proposed several nonparametric estimators for the tail copula and for the tail dependence coefficient. They proved their weak convergence, asymptotic normality, and strong consistency. Hsing et al. (2004) defined the bivariate tail dependence function which includes as a particular case the tail dependence coefficient, and introduced a method to visualize extreme tail dependence. Schmidt (2003) characterized tail dependence within the class of elliptical and extreme value copulae, and provided a parametric and non-parametric estimator for the tail dependence coefficient. Frahm et al. (2005) presented several parametric and nonparametric estimators for the tail dependence coefficient. In a detailed simulation study, they compared the advantages and disadvantages of all estimators.

\subsection{Simulating from the GT-copula}

Consider the stochastic representation (15) of a $d$-dimensional random vector $\boldsymbol{X}$ with distribution $\operatorname{MGT}(\mu, \Sigma, \lambda, \beta, q)$. An algorithm to generate data from the $d$-dimensional MGT distribution and GT-copula may be as follows:

- Find the Cholesky decomposition $A$ of $R$, where $R$ is the correlation matrix;

- Simulate a random variate $u^{(d)}$ from a uniform distribution in $R^{d}$;

- Simulate a random variate $v$ from the generalized beta distribution of second kind and independent of $u^{(d)}$;

- Set $\mathbf{y}=A^{\prime} u^{(d)}$. Note that this is a $d$-dimensional vector;

- Set $\mathbf{x}=\mu+v \mathbf{y}$. Note that $\mathbf{x}$ has a $d$-dimensional MGT-distribution;

- Set $u_{i}=Q\left(x_{i}\right), i=1, \ldots, d$.

The resulting $\left(u_{1}, \ldots, u_{d}\right)^{\prime}$ is a random variate from a $d$-dimensional GT-copula.

\section{Examples of Multivariate Skew Distributions}

In this section we use the GT-copula to define a new family of multivariate skew distributions. Our main focus is the asymmetric, possibly multimodal case, which is not covered by other existing skew multivariate distributions. The idea is as follows. Consider the random vector $\boldsymbol{X}$ with each component $X_{i}, i=1, \ldots, d$, having continuous density $f_{i}\left(x_{i}\right)$ and corresponding cdf $F_{i}\left(x_{i}\right)$. Let the random 
vector $\boldsymbol{Z}$ be $\operatorname{MGT}(0, R, 1, \beta, q)$, where $R=\left\{\rho_{i j}: \rho_{i i}=1,-1<\rho_{i j}<1\right.$ for $i \neq$ $\left.j, \rho_{i j}=\rho_{j i} ; i, j=1, \ldots, d\right\}, R$ a positive definite matrix. Suppose that $Z_{i}=$ $Q^{-1}\left(F_{i}\left(X_{i}\right)\right)$, for $i=1, . ., d$. Then the joint density function of $\boldsymbol{X}$ can be obtained as

$$
h\left(x_{1}, \ldots x_{d}\right)=c_{G T}^{R, \nu}\left(F_{1}\left(x_{1}\right), \ldots, F_{d}\left(x_{d}\right)\right) \prod_{i=1}^{d} f_{i}\left(x_{i}\right)
$$

Distributions constructed in this way have been named by Fang et al. (2002) as meta-elliptical distributions. The random vectors $\boldsymbol{X}$ and $\boldsymbol{Z}$ possess the same copula, termed meta-elliptical by Abdous et al. (2005).

Note that in (26) one can choose any marginal distribution. All multivariate distributions (26) will have the same copula-based measures, for example, Kendall's $\tau$ coefficient, which only depends on $C_{G T}^{R, \nu}$. Also note that the variables $X_{i}, X_{j}$, will be positively or negatively correlated according to $\rho_{i j} \geq 0$ or $\rho_{i j} \leq 0$, and moreover, despite the choices of $F_{i}, i=1,2$, by letting $\rho_{i j} \rightarrow+1$ or $\rho_{i j} \rightarrow-1$, the margins become perfectly correlated.

It would be interesting to interpret the dependence matrix $R$ in the new multivariate distributions (see Song (2000)). By construction, $\rho_{i j}=\operatorname{corr}\left(Z_{i}, Z_{j}\right)=$ $\operatorname{corr}\left(Q^{-1}\left(F_{i}\left(X_{i}\right)\right), Q^{-1}\left(F_{j}\left(X_{j}\right)\right)\right)$. That is, the $\rho_{i j}$ s (the Pearson linear correlation coefficient of the original variables $\left.\left(Z_{1}, \ldots, Z_{d}\right)\right)$ are now the the Pearson correlation coefficient of nonlinear transformations of the $\left(X_{1}, \ldots, X_{d}\right)$. Thus, in the new multivariate distributions, $R$ contains pairwise measures of nonlinear association between the variables. Note that if $R$ is the identity matrix, then $\left(X_{1}, \ldots, X_{d}\right)$ are uncorrelated. In addition, note that the tail dependence concerns the copula by itself.

To obtain asymmetric multivariate distributions we use skew marginal distributions. We especially focus on skew margins possessing different degrees and directions of skewness. To illustrate that, we consider the univariate skew GT Distribution proposed in Theodossiou (1998), which has density function

$$
f(x, k, \nu, \lambda)=\left[\begin{array}{ll}
M\left(1+\frac{k}{\nu-2} \frac{|x|^{k}}{\theta^{k}(1-\lambda)^{k}}\right)^{-(\nu+1) / k} & \text { for } x<0 \\
M\left(1+\frac{k}{\nu-2} \frac{|x|^{k}}{\theta^{k}(1+\lambda)^{k}}\right)^{-(\nu+1) / k} & \text { for } x \geq 0
\end{array}\right.
$$

where $k, \nu$ and $\lambda$ are the scaling parameters and $M=0.5 k B(1 / k, \nu / k)^{-3 / 2} B(3 / k$, $(\nu-2) / k)^{1 / 2} S(\lambda)$ is the normalizing constant ensuring that the above density function is a proper density function. $\mathrm{B}(\cdot, \cdot)$ is the beta function, $\theta=(k /(\nu-2))^{1 / k}$ $B(1 / k, \nu / k)^{1 / 2} B(3 / k,(\nu-2) / k)^{-1 / 2} S(\lambda)^{-1}$, and $S(\lambda)=\left(1+3 \lambda^{2}-4 \lambda^{2} B(2 / k,(\nu-\right.$ $\left.1) / k)^{2} B(1 / k, \nu / k)^{-1} B(3 / k,(\nu-2) / k)^{-1}\right)^{1 / 2}$. This family of skew distributions includes the following special cases: 
- The case $\lambda=0$ gives symmetric univariate GT Distribution proposed by McDonald and Newey (1988) with density function

$$
f(x, k, \nu)=\frac{k}{2 \nu^{1 / k} B(1 / k, \nu)}\left(1+\frac{|x|^{k}}{\nu}\right)^{-(\nu+1) / k}
$$

- The case $\lambda=0$ and $k=2$ gives the $t$ distribution.

- The case $k=2$ gives the skew $t$ distribution proposed by Hansen (1994).

Figure 1 shows an example of a family of skew meta-elliptical distributions constructed from the GT-copula. This figure shows the density perspective (left) and the density contours (right) of the particular case of the multivariate distribution (26), obtained by setting $d=2$ and choosing a GT-copula with $\beta=1, \nu=4$, $\rho=0.5$. The margins are composed by the asymmetric distributions (27), with $k=2, \nu=4$, and $\lambda$ equal to 0.4 and 0.4 for the upper figures, and $\lambda$ equal to 0.7 and 0.4 for the bottom figures. 

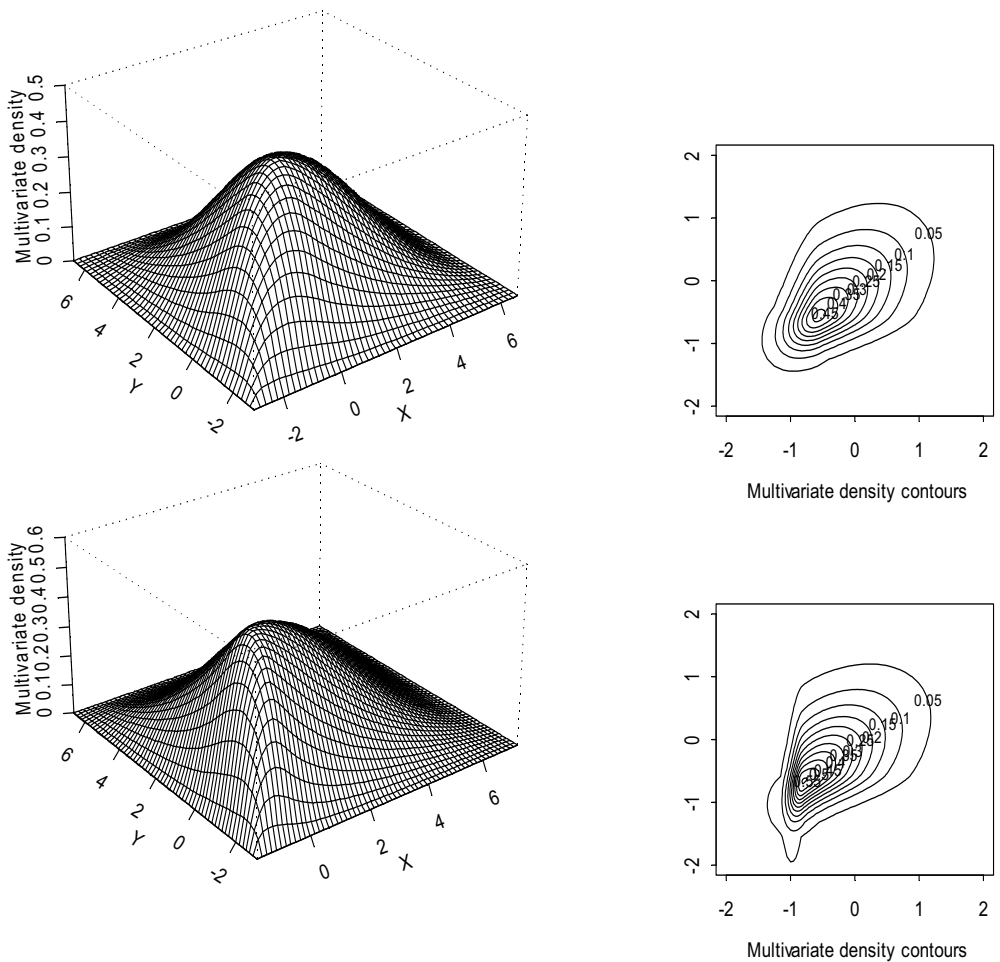

Figure 1

Density perspective (left) and contours (right) of a meta-elliptical distribution constructed from a GT-copula with $\beta=1, \nu=4, \rho=0.5$, and asymmetric $t$-distributed margins with $k=2, \nu=4$, and $\lambda$ equal to 0.4 and 0.4 for the upper figures, and $\lambda$ equal to 0.7 and 0.4 for the bottom figures 


\section{Measuring Financial Market Interdependencies}

The bottom line in many applications in finance is the multivariate modeling of a set of risky assets. Usually, the challenge is to estimate the dependence structure required for developing methods for hedging risk, for asset classification, portfolio optimization, and also to compute alternative measures of dependence. In this section we illustrate the potentiality of copula modeling in finance by working on a simple exercise using log-returns.

Squared $\log$ returns usually show significant autocorrelation in the first few lags, although daily log-returns are not in general significantly autocorrelated. This motivates us to analyze the data under two different approaches. First, we treat the (raw) returns as if they formed a random sample. In a second exercise, we fit ARMA-GARCH type models to the returns to remove the dynamics in the mean and in the volatility, and then analyze the filtered returns. We should note that filtering the data through GARCH type models is not a monotonic transformation. Thus, we do not expect the raw and filtered log daily returns to possess the same copula.

Let $X_{1}$ and $X_{2}$ denote financial returns from markets 1 and 2. Whenever there exists any type of positive dependence among the markets, the probability

$$
\operatorname{Pr}\left\{X_{1}>x_{1} \mid X_{2}>x_{2}\right\}
$$

is greater than $\operatorname{Pr}\left\{X_{1}>x_{1}\right\}$, property also known as the positive quadrant dependence, introduced by Lehmann (1966). In this case we say that there exists contagion between markets 1 and 2 (see definitions of contagion in Straetmans et al. (2004) and in the references therein). This interdependence may change during crisis and is not necessarily explained by economic theories. ${ }^{1}$

The correlation coefficient and its conditional version have been widely used in empirical analysis of dependence across financial markets. However, it has been shown (Boyer et al. (1999), Longin and Solnik (2001), Embrechts et al. (2002)) that they can be misleading. As shown in Section 2, two copulas may have the same value for the correlation coefficient, but just one of them can capture an extra type of nonlinear dependence.

To assess the strength of the linkage between markets during stress periods, we compute the probabilities (29) and choose as meaningful values $x_{1}$ and $x_{2}$, the unconditional model based Value-at-Risk with small exceedance probability $\alpha$ in each margin, respectively denoted as the $\operatorname{VaR}\left(X_{1}, \alpha\right)$ and the $\operatorname{VaR}\left(X_{2}, \alpha\right)$.

Rewriting (29) in terms of copulas, we assess linkages at extreme levels by computing

\footnotetext{
${ }^{1}$ For example, markets with similar economic or political positions to another one experiencing crisis, may be seen with precaution from market participants, even when the countries involved have weak trade links.
} 


$$
\frac{1-u-u+C(u, u)}{1-u}
$$

where $u=F_{1}\left(\operatorname{VaR}\left(X_{1}, \alpha\right)\right)=F_{2}\left(\operatorname{VaR}\left(X_{2}, \alpha\right)\right)=1-\alpha$. Note that under independence (30) is equal to $1-u$, and that this holds at $\rho=0$ only for Gaussian (meta-)elliptical copulas, as established in Abdous et al. (2005). Also, the stronger the dependence structure between the two markets (as modeled by $C$ ), the larger (30) is.

According to the results of Section 2, expression (30) is the function $\lambda_{U}(\alpha)$, whose limit as $\alpha \rightarrow 0^{+}$is the upper tail dependence coefficient $\lambda_{U}$. We may then interpret $\lambda_{U}$ as a measure of asymptotic linkage. As we have seen, the Gaussian copula has $\lambda_{U}$ equal to zero, but the $t$ - and the GT-copulas possess non-zero tail dependence. ${ }^{2}$ For the illustration that follows, we compute the function $\lambda(\alpha)$ $\left(\lambda(\alpha)=\lambda_{U}(\alpha)=\lambda_{L}(\alpha)\right)$ for a decreasing sequence of values $\alpha$.

To illustrate that we use a pair of stock market indexes: The Dow Jones Industrial Average (U. S.) and the IBOVESPA (Brazil). Data are from Economatica and consist of daily log-returns from January/1995 to June/2003. The copulas considered are the Gaussian, the $t$ - and GT-copulas. We apply the maximum likelihood estimation method in two steps, the so-called IFM method (inference function for margins, Joe (1997)). For some models the efficiency of this procedure has been established, see Xu (1996), Joe (1997), and discussion in Frahm et al. (2005).

Under the first approach for modeling the margins, we fit the Normal, the asymmetric $t$ of Hansen (1994), and the symmetric $t$ distributions to the sample $\left.\left(\left(x_{1,1}, x_{1,2}\right)\right), \ldots,\left(x_{T, 1}, x_{T, 2}\right)\right)$ of log-return observations. For each margin $i=1,2$, the best fit $\hat{F}_{i}$ is chosen based on standard goodness-of-fit tests. In the second step of the IFM method, the copula parameters are estimated from the approximate realizations $\left(u_{t, 1}, u_{t, 2}\right)=\left(\widehat{F}_{1}\left(x_{t, 1}\right), \widehat{F_{2}}\left(x_{t, 2}\right)\right), t=1, \ldots, T$. The best copula fit is chosen based on the $A I C$ criterion, and then a goodness-of-fit test is applied based on a bivariate extension of the usual Pearson chi-square test, described in Genest and Rivest (1993). ${ }^{3}$

Under the second approach, we assume that, for $i=1,2, x_{t, i}=\mu_{t, i}+\sigma_{t, i} y_{t, i}$, where $\mu_{t, i}$ is defined through some ARMA model, $\sigma_{t, i}$ is specified by some GARCH model, and $y_{t, i}, t=1, \ldots, T$ are independent and identically distributed random variables with zero mean, unit variance, and marginal distribution $F_{i}$, either a Gaussian or a $t$-Student. The ARMA-GARCH models are estimated by maxi-

\footnotetext{
${ }^{2}$ However, caution is needed in the case of asymptotic independence, since the convergence of $\lambda(\alpha)$ to zero may be very slow (Coles et al. (1999), Costinot et al. (2000)) As we will see in the application that follows, even for copulas with very small $\lambda_{U}$, the interdependencies may be relevant for small probabilities $\alpha$.

${ }^{3}$ We note that there are other goodness-of-fit techniques used to assess the quality of the models. In the present context, for example, methods developed in Wang and Wells (2000), Fermanian (2005) or Chen and Fan (2005), among others, could be implemented.
} 
mum likelihood, and the best model is chosen based on the AIC criterion. The filtered log-returns $\left(\widehat{y_{t, 1}}, \widehat{y_{t, 2}}\right)=\left(\frac{x_{t, 1}-\widehat{\mu_{t, 1}}}{\widehat{\sigma_{t, 1}}}, \frac{x_{t, 2}-\widehat{\mu_{t, 2}}}{\widehat{\sigma_{t, 2}}}\right)$, free of serial dependence and volatility clusters, are used to finally obtain the copula approximate realizations $\left(u_{t, 1}, u_{t, 2}\right)=\left(F_{1}\left(\widehat{y_{t, 1}}\right), F_{2}\left(\widehat{y_{t, 2}}\right)\right), t=1, \ldots, T$.

Table 1

Lower left and upper right quadrants linkage estimates for the pair U.S. and Brazil

\begin{tabular}{l|c|c|c|c}
\hline & $\widehat{\lambda}(0.10)$ & $\widehat{\lambda}(0.05)$ & $\widehat{\lambda}(0.01)$ & $\widehat{\lambda_{U}}=\widehat{\lambda_{L}}$ \\
\hline Log-returns & $0.3040(0.05)$ & $0.2072(0.04)$ & $0.1236(0.02)$ & $0.000028(0.000009)$ \\
\hline Independence & $0.1000(0.02)$ & $0.0500(0.01)$ & $0.0100(0.003)$ & $0.000000(0.0)$ \\
\hline Filtered data & $0.3440(0.04)$ & $0.2644(0.06)$ & $0.1273(0.02)$ & $0.024810(0.0067)$ \\
\hline
\end{tabular}

The best univariate fits for the raw log-returns of the Dow Jones and the IBOVESPA were, respectively, the symmetric $t$ ( 5 d.f.) and the asymmetric $t$ $(\lambda=0.06$ and 4 d.f.) distributions. The best copula fit turned out to be a GT-copula with $\rho=0.45, \beta=0.967$, and 56 d.f. We provide the estimates of $\lambda(\alpha)$ and their standard errors in the first row of Table 1. Due to the copula symmetry, results for the bull and bear markets are the same. Even though the value $\lambda_{U}=0.000028$ is really small, the linkages (30) at high quantiles (on the copula diagonal) are quite strong. For example, for the small probability 0.01, we found $\lambda(0.01)=0.1236$, see Table 1 . This means that the probability that one of the markets goes beyond its $\operatorname{VaR}(\cdot, 1 \%)$, given that the other one had broken through its $\operatorname{VaR}(\cdot, 1 \%)$, is more than 10 times greater than its value under independence $(1 \%$, second row of Table 1$)$.

We now choose different cutoff values $x_{1}$ and $x_{2}$ and consider

$$
\operatorname{Pr}\left\{X_{1}>x_{1} \mid X_{2}>x_{2}\right\}=\frac{1-u-v+C(u, v)}{1-v}
$$

where, for example, $u=0.99, v=0.95$, and therefore $x_{1}=\operatorname{VaR}\left(X_{1}, 1 \%\right)$ and $x_{2}=$ $\operatorname{VaR}\left(X_{2}, 5 \%\right)$. This is the probability of market 1 going beyond its $\operatorname{VaR}(\cdot, 1 \%)$, given that market 2 has just surpassed its $\operatorname{VaR}(\cdot, 5 \%)$. Let $x_{1}^{10}, x_{1}^{5}$, and $x_{1}^{1}$, and $x_{2}^{10}, x_{2}^{5}$, and $x_{2}^{1}$, denote, respectively the VaR of $X_{1}$ and $X_{2}$ with exceedance probabilities $1-u=1-v=10 \%, 5 \%$, and $1 \%$. Using the fitted univariate models, we found the VaR values for the pair U.S. (1) and Brazil (2) to be, respectively, equal to $(1.421,1.916,3.155)$ and $(3.015,4.145,7.191)$.

In Table 2 we give the probabilities associated with a selection of tail events involving the VaR values of markets (1) and (2). These numbers may provide insights into the interrelationships of these markets. We start by considering the sequence of probabilities

$$
\operatorname{Pr}\left\{X_{1}>x_{1}^{5} \mid X_{2}>x_{2}^{10}\right\} \quad, \quad \operatorname{Pr}\left\{X_{1}>x_{1}^{1} \mid X_{2}>x_{2}^{10}\right\}
$$


which measure tail events in the same conditional probability space. From the copula symmetry, the probabilities (31) are equal for the lower-left and the upperright quadrants. For the pair U.S. and Brazil, they are given in the 1st row, and in columns 2 and 3 of Table 2 (0.1816 and 0.0974). They should be contrasted against the unconditional probabilities 0.05 and 0.01 (see 2nd row). From the examination of columns 2 and 4 of Table 2 , it seems that contagion increases as both markets get into stress periods.

This suggests the investigation of the probability of two markets crashing together, given that at least one of them is already in crisis (see Hartman et al. (2001) and Straetmans (1999)), given by

$$
\operatorname{Pr}\left\{X_{2}>x_{2}, X_{1}>x_{1}^{1} \mid X_{2}>x_{2} \text { or } X_{1}>x_{1}^{1}\right\}
$$

for some large value $x_{2}, x_{2}=F_{2}^{-1}(v)$. This means computing the limit as $v \rightarrow 1$ of

$$
\begin{aligned}
\operatorname{Pr}\left\{X_{2}\right. & \left.>F_{2}^{-1}(v), X_{1}>F_{1}^{-1}(u) \mid X_{2}>F_{2}^{-1}(v) \text { or } X_{1}>F_{1}^{-1}(u)\right\} \\
& =\frac{1-u-v+C(u, v)}{1-C(u, v)}
\end{aligned}
$$

for fixed $u=0.99$. For example, for $1-v=0.01$ we obtained 0.08342. As a measure of risk (or contagion) one could compare this figure to the unconditional probability 0.0012 . It is important to stress that all these probabilities depend upon the marginals only through the VaR values.

We now take the second approach and fit the copula models to the approximate realizations $\left(u_{t, 1}, u_{t, 2}\right)=\left(F_{1}\left(\widehat{y_{t, 1}}\right), F_{2}\left(\widehat{y_{t, 2}}\right)\right), t=1, \ldots, T$, based on the filtered log-returns. For the pair U.S. and Brazil, the best model turned out to be a combination of an $\operatorname{ARMA}(1,1)$ for the conditional mean, and a $\operatorname{GARCH}(1,1)$ model with leverage term with $F_{i}$ being a t-Student distribution with 8 and 11 d.f. respectively for $i=1,2$. The best copula fit was the GT-copula with $\rho=0.47$, $\beta=0.99$, and 16 d.f. Tables 1 and 2 , in the third row, provide the probabilities associated with the dependence structure of the filtered log-returns. We found these probabilities to be now slightly bigger.

As already commented, raw and filtered log-returns are not expected to possess the same copula. For this specific illustration, we found weaker dependence for the raw log-returns. These considerations call for a much more comprehensive empirical work and theoretical investigations. 
Table 2

Probabilities of tail events for the pair U.S. $\left(X_{1}\right)$ and Brazil $\left(X_{2}\right)$

\begin{tabular}{l|c|c|c}
\hline & {$\left[X_{1}>x_{1}^{5} \mid X_{2}>x_{2}^{10}\right]$} & {$\left[X_{1}>x_{1}^{1} \mid X_{2}>x_{2}^{10}\right]$} & {$\left[X_{1}>x_{1}^{1} \mid X_{2}>x_{2}^{5}\right]$} \\
\hline Raw log-returns & 0.1816 & 0.0974 & 0.1152 \\
\hline Independence & 0.0500 & 0.0100 & 0.0100 \\
\hline Filtered log-returns & 0.2108 & 0.1014 & 0.1324 \\
\hline Notation in table: $[A \mid B]$ denotes event $A$ given $B$
\end{tabular}

\section{Conclusions}

In this paper we obtained the GT-copula pertaining to the multivariate GT distributions and derived some of its properties, including its coefficient of tail dependence. The advantage of the GT-copula over the existing elliptical copulas, such as the Gaussian or the $t$-copula, is its extra parameter, which allows for more flexibility when fitting data. We showed how to construct a new general class of skew meta-elliptical distributions using Sklar's theorem and provided some examples built on marginal skew t-distributions. As an illustration of its applicability we provided a simple exercise in finance. Using the fitted copula we computed several measures of stock market linkages, and computed changes in interdependencies at extreme levels. We also examined the effect of volatility filtering on copula and contagion estimates.

Inference was based on the maximum likelihood method in two steps: margins first and then copula models. In the illustration we provided, the univariate fits posed no difficulties. Choosing a parametric copula family for a particular application is a crucial issue. Elliptical copulas are a natural extension of the multivariate normal model and possess nice properties. All elliptical copulas fitted yielded very close estimates for $\rho$. The robustness of the $\rho$ estimate with respect to copula models validates the $\rho$ value found as a real indication of dependence.

Important issues such as modeling based on time-varying copulas, or the development of fast algorithms for copula estimation, were not addressed in the real data application of this paper. Efficient algorithms for fitting elliptical copulas to data, in particular the $t$ - or the GT, are still an open research problem. In the application provided, our focus was on the dissemination of copula-related concepts, emphasizing their importance in the area of finance. We hope to have motivated practitioners to get acquainted with this subject and to try their own applications.

There are ways of introducing skewness into the copula itself. This could be obtained by working on the many generalizations of the multivariate $t$ distribution presented in the literature at large, some of which allow skewness, offering the potential for asymmetric copulas. In a further work this may be developed in order to place the GT-copula or the MGT distribution in the larger context of skew multivariate $t$ distributions. 


\section{References}

Abdous, B., Genest, C., \& Rémillard, B. (2005). Dependence properties of metaelliptical distributions. In Duchesne, P. \& Rémillard, B., editors, Statistical Modeling and Analysis for Complex Data Problems, pages 1-15. Kluwer, Dordrecht, The Netherlands.

Arellano-Valle, R. B. \& Bolfarine, H. (1995). On some characterizations of the t distribution. Statistics and Probability Letters, 25:79-85.

Arslan, O. (2004). Family of multivariate generalized t-distribution. Journal of Multivariate Analysis, 89:329-337.

Boyer, B., Gibson, M., \& Mulder, C. (1999). Pitfalls in tests for changes in correlations. Board of Governors of the Federal Reserve System, International Finance Discussion Paper, 597.

Chen, X. \& Fan, Y. (2005). Pseudo-likelihood ratio tests for semiparametric multivariate copula model selection. The Canadian Journal of Statistics, 33(3):389414.

Coles, S., Currie, J., \& Tawn, J. (1999). Dependence measures for extreme value analysis. Department of Mathematics and Statistics, Lancaster University, Working Paper.

Costinot, A., Roncalli, T., \& Teiletche, J. (2000). Revisiting the dependence between financial markets with copulas. Working Paper.

Demarta, S. \& McNeill, A. J. (2004). The t-copula and related copulas. International Statistical Review, 73(1):111-129.

Embrechts, P., McNeil, A., \& Straumann, D. (2002). Correlation and dependency in risk management: Properties and pitfalls. In Dempster, M. A. H., editor, Risk Management: Value at Risk and Beyond, pages 176-223. Cambridge University Press, Cambridge.

Fang, H., Fang, K.-T., \& Kotz, S. (2005). Corrigendum to the meta-elliptical distribution with given marginal. Journal of Multivariate Analysis, 94:222-223.

Fang, H.-B., Fang, K.-T., \& Kotz, S. (2002). The meta-elliptical distributions with given marginals. Journal of Multivariant Analysis, 82:1-16.

Fang, K.-T., Kotz, S., \& Ng, K. W. (1990). Symmetric Multivariate and Related Distributions. Chapman and Hall, London.

Fermanian, J.-D. (2005). Goodness-of-fit tests for copulas. Journal of Multivariate Analysis, 2:119-152. 
Frahm, G., Junker, M., \& Schmidt, R. (2005). Estimating the tail-dependence coefficient: Properties and pitfalls. Insurance: Mathematics and Economics, 37:80-100.

Frahm, G., Junker, M., \& Szimayer, A. (2003). Elliptical copulas: Applicability and limitations. Statistics and Probability Letters, 63(3):275-286.

Genest, C. \& Rivest, L.-P. (1993). Statistical inference procedures for bivariate Archimedean copulas. Journal of the American Statistical Association, 88:10341043.

Hansen, B. E. (1994). Autoregressive conditional density estimation. International Economic Review, 35(3):705-730.

Hartman, P., Straetmans, S., \& De Vries, C. G. (2001). Asset market linkages in crisis periods. Working Paper 71, European Central Bank.

Hsing, T., Klüppelberg, C., \& Khun, G. (2004). Modelling estimation and visualization of multivariate dependence for risk management. Available at ttp://www-m4.ma.tum.de/ Papers/index.en.html.

Hult, H. \& Lindskog, F. (2002). Multivariate extremes, aggregation and dependence in elliptical distributions. Advances in Applied Probability, 34(3):587-608.

Joag-Dev, K., Perlman, M. D., \& Pitt, L. D. (1983). Association of normal random variables and Slepian's inequality. Annals of Probability, 11:451-455.

Joe, H. (1997). Multivariate Models and Dependence Concepts. Chapman and Hall, London.

Jouini, M. N. \& Clemen, R. T. (1996). Copula models for aggregating expert opinions. Operations Research, 44(3):444-457.

Lehmann, E. L. (1966). Some concepts of dependence. The Annals of Mathematical Statistics, 37:1137-1153.

Longin, F. \& Solnik, B. (2001). Correlation structure of international equity markets during extremely volatile periods. CEPR, Discussion Paper, 2538.

McDonald, J. B. \& Newey, W. K. (1988). Partially adaptive estimation of regression models via the generalized $\mathrm{t}$ distribution. Journal of Economic Theory, 4:429-457.

Nelsen, R. B. (1999). An Introduction to Copulas, volume 139. Springer Verlag, New York. Lecture Notes in Statistics, 139,.

Schmidt, R. (2002). Tail dependence for elliptically contoured distributions. Mathematical Methods of Operations Research, 55:301-327. 
Schmidt, R. (2003). Credit risk modelling and estimation via elliptical copulae. In Bohl, G., Nakhaeizadeh, G., Rachev, S. T., Ridder, T., \& Vollmer, K. H., editors, Credit Risk - Measurement, Evaluation and Management, pages 267289. Physica-Verlag, Heidelberg.

Schmidt, R. \& Stadtmüller, U. (2003). Nonparametric estimation of tail dependence. London School of Economics and University of Ulm. Submitted to Blackwell Publishing, December 2003.

Sklar, A. (1959). Fonctions de repartition à n dimensions et leurs marges. Publications de l'Institut de Statistique de l'Université de Paris, 8:229-231.

Song, P. X. (2000). Multivariate dispersion models generated rom Gaussian copula. Scandinavian Journal Statistics, 27:305-320.

Straetmans, S. (1999). Extreme financial returns and their comovements. Tinbergen Institute Research Series, 181. Erasmus University Rotterdam's Thesis.

Straetmans, S., de Vries, C. G., \& Hartmann, P. (2004). Asset market linkages in crisis periods. Review of Economics and Statistics, 86(1):313-326.

Tawn, J. (1988). Bivariate extreme value theory: Models and estimation. Biometrika, 75(3):397-415.

Theodossiou, P. (1998). Financial data and the skewed generalized t distribution. Management Science, Part 1(12):1650-1661.

Wang, W. \& Wells, M. T. (2000). Model selection and semiparametric inference for bivariate failure-time data. Journal of the American Statistical Association, $2(2): 73-85$.

Wendin, J. (2002). Estimation of the Spectral Measure and the Tail Dependence Coefficient for Regularly Varying Random Vectors. PhD thesis, ETH, Zürich.

Xu, J. J. (1996). Statistical Modeling and Inference for Multivariate and Longitudinal Discrete Response Data. PhD thesis, Dept. of Statistics, University British Columbia. 